\title{
DORMIR, COMER Y HABLAR: VÍNCULO SIMBÓLICO
}

\section{ARTÍ́CULO ORIGINAL}

PALLADINO, Ruth Ramalho Ruivo1, SOUZA, Luiz Augusto de Paula², PALLOTTA, Mara Lucia ${ }^{3}$, COSTA, Rogério da ${ }^{4}$, CUNHA, Maria Claudia ${ }^{5}$

PALLADINO, Ruth Ramalho Ruivo. Et al. Dormir, comer y hablar: vínculo simbólico. Revista Científica Multidisciplinar Núcleo do Conhecimento. Año 06, Ed. 08, Vol. 06, págs. 153-170. Agosto 2021. ISSN: 2448-0959, Enlace de acceso: https://www.nucleodoconhecimento.com.br/psicologia-es/comer-y-hablar, $\quad$ DOI: 10.32749/nucleodoconhecimento.com.br/psicologia-es/comer-y-hablar

\section{RESUMEN}

El sueño, la comida y el lenguaje son pilares de la vida saludable de los niños, se entrelazan desde el nacimiento y conforman la estructura dinámica del desarrollo infantil. Estos son los efectos de las condiciones interdependientes: orgánicas, psíquicas y sociales, que involucran al niño y resultan, simultáneamente, de herencias orgánicas y simbólicas. Este último sobredetermina y modula la interacción del niño con el entorno, especialmente con el otro humano que está allí. Esta herencia dibujará patrones de conducta y comportamiento que a menudo pueden contribuir a cambios que comprometen, hasta cierto punto, el desarrollo general del niño. En la clínica infantil, la descripción de los trastornos del desarrollo, desde los más leves hasta los más graves, incluye, por regla general, aspectos de

\footnotetext{
${ }^{1}$ Doctor en Psicología Clínica, Profesor doctor del Programa de Posgrado en Comunicación Humana y Salud, Facultad de Humanidades y Salud, PUC-SP (ORCID: https://orcid.org/0000-0001-8466-838X).

${ }^{2}$ Doctor en Psicología Clínica, profesor titular del Programa de Posgrado en Comunicación Humana y Salud, Facultad de Humanidades y Salud, PUC-SP (ORCID: https://orcid.org/0000-0003-4968-9753).

${ }^{3}$ Estudiante de doctorado en Comunicación Humana y Salud en la PUC-SP (ORCID: https://orcid.org/00000001-5986-9657).

${ }^{4}$ Doctor en Filosofía, Profesor de Doctorado del Programa de Posgrado en Comunicación y Semiótica, PUC-SP (ORCID: https://orcid.org/0000-0002-6807-4263).

${ }^{5}$ Doctor en Psicología Clínica, profesor titular del Programa de Posgrado en Comunicación Humana y Salud, Facultad de Humanidades y Salud, PUC-SP (ORCID: https://orcid.org/0000-0003-3198-6995).
}

RC: 98234

Disponible: https://www.nucleodoconhecimento.com.br/psicologia-es/comer-y-hablar 
alimentación, sueño y lenguaje, lo que sugiere, entonces, una tríada de base, cuestionando a los médicos sobre la posibilidad de que exista, más que una simple coincidencia, una correlación entre las funciones biológicas fundamentales. Si este es el caso, será importante que el clínico se apropie de esta perspectiva, ya que la implicación probablemente determinará particularidades en los procedimientos de diagnóstico y tratamiento. En esta dirección, vale la pena profundizar y discutir el desarrollo de estas funciones (sueño, dieta, lenguaje), buscando aclarar su correlación constitutiva, el vínculo entre ellas.

Palabras clave: Idioma, Alimentación, Sueño.

\section{INTRODUCCIÓN}

El sueño, la comida y el lenguaje son pilares de la vida saludable de los niños, se entrelazan desde el nacimiento y conforman la estructura dinámica del desarrollo infantil.

Tal entrelazamiento, sin embargo, no es un postulado unánime, en los proyectos para la descripción y comprensión de esta tríada o cada parte se toma por separado o, entonces, se señala el privilegio de una parte sobre la otra, es decir, serían relaciones que no pueden definirse como implicación.

Para pensar en la implicación es necesario asumir que dormir, comer y hablar involucran al cuerpo, pero un cuerpo que exige un nombre, un cuerpo subjetivo y, por lo tanto, un cuerpo alistado por lo simbólico: en la vinculación del nombre propio y el cuerpo hay, en la lectura de este rasgo, algo que se articula a través de la apropiación, de sí mismo, que no es un elemento tan obvio y simple en la constitución humana (LEITE, 2008, p.16). ¿Cómo se representa tal apropiación del cuerpo, que proviene de su propio cuerpo cuando es nombrado? Nombrar el cuerpo es reconocerlo en el campo simbólico, efecto de infinitas articulaciones, acciones y entrelazar.

RC: 98234

Disponible: https://www.nucleodoconhecimento.com.br/psicologia-es/comer-y-hablar 
Los patrones de sueño, alimentación y habla son efectos de condiciones interdependientes: orgánicas, psíquicas y sociales, que involucran al niño y resultan, simultáneamente, de herencias orgánicas y simbólicas. Este último sobredetermina y modula la interacción del niño con el entorno, especialmente con el otro humano que está allí.

Para los bebés, este entorno puede estar representado, privilegiadamente, por la figura materna, la madre y, vale la pena señalar, ni siquiera es necesariamente la madre biológica o los cuidadores, sino una instancia materna asana, otro humano que inscribe el "cercano-que-ayuda", el nebensmench freudiano, como explica Cabassu (2003). Aquel que, más que garantizar la supervivencia del organismo del niño, lo reconoce como sujeto, constituye el vínculo entre deseo (materno) y palabra, a partir del cual la subjetividad y las relaciones sociales se hacen posibles y comienzan para el bebé, nombrando su cuerpo y su inscripción en un texto de afiliación y sociabilidad.

Si, como decíamos, el sueño, la alimentación y el lenguaje son pilares de la vida del niño, su implicación depende, por tanto, de las expresiones maternas dirigidas a él, pero sólo si - para saciar el hambre, calentarse del frío, empacar para dormir... van acompañados de un deseo no anónimo (STORK, LY, MOTA, 1997, p. 34): esta es la presencia del otro, la institución de la relación materna.

Además, es importante saber que es necesario reconocer en las actitudes maternas un sentido cultural, igualmente determinante, porque las madres interpretan $y$ responden a las manifestaciones [...] del bebé según las normas de la sociedad a la que pertenecen, incluso si sus respuestas también están moduladas por la dinámica psíquica personal (CISMARESCO, 1997, p. 267).

Esta condición presupone, más ampliamente, la centralidad de la función de una familia continente, para lo cual el concepto "Yo-piel" de Anzieu (1989) puede ser esclarecedor, ya que se refiere a los límites entre lo biológico y lo simbólico y, para el bebé, su establecimiento responde a la necesidad de una envoltura narística y

RC: 98234

Disponible: https://www.nucleodoconhecimento.com.br/psicologia-es/comer-y-hablar 
asegura al aparato psíquico la certeza y constancia de un bienestar básico (op. cit., p. 44). Este sentimiento de seguridad será fundamental para el sentimiento de pertenencia, básico para la construcción de la identidad del niño.

Esta herencia, a la vez orgánica y simbólica, transmitida en las relaciones del niño con el otro, aliada -por supuesto- a la herencia orgánica, dibujará patrones de conducta y comportamiento que muchas veces pueden contribuir a cambios que comprometan, en cierta medida, el desarrollo general del niño.

En la clínica infantil, la descripción de los trastornos del desarrollo, desde los más leves hasta los más graves, incluye, por regla general, aspectos de alimentación y sueño (WINNICOTT, 1975; MADEIRA, AQUINO, 2003 ; SANTOS, 2004; JERUSALINSKY, 2004).

El hecho de que estos síntomas estén casi siempre alineados entre sí, cuestiona a los médicos sobre la posibilidad de que exista, más que una simple coincidencia, una correlación entre las funciones biológicas fundamentales.

Además, en las descripciones clínicas de los trastornos del desarrollo del lenguaje, los informes de problemas de alimentación (PALLADINO, CUNHA, SOUZA, 2007) y el sueño son frecuentes, lo que sugiere, entonces, una tríada de correlaciones.

Sin embargo, y a menudo, los diferentes campos de estudio del desarrollo no comparten la idea de una correlación significativa entre tales funciones, y es común que los problemas de sueño, alimentación y lenguaje se piensen por separado. Cuando este es el caso, en presencia de cambios en el desarrollo, el fenómeno se asume como comorbilidad.

Hay excepciones y tal predominio, es un hecho, con relevancia para el psicoanálisis y ciertos enfoques de la psicología (GROMANN, 2002), así como para la pequeña porción de estudios de disciplinas que dialogan con ambas, como la logopedia, la

RC: 98234

Disponible: https://www.nucleodoconhecimento.com.br/psicologia-es/comer-y-hablar 
medicina psiquiátrica, la neurología y la endocrinología. Estas excepciones inspiraron una mayor reflexión.

Asumir las correlaciones entre las funciones permite aclarar la alineación sintomatológica observada entre los síntomas de alimentación, sueño y lenguaje en la primera infancia. Si este es el caso, será importante que el clínico se apropie de esta perspectiva, ya que la implicación probablemente determinará particularidades en los procedimientos de diagnóstico y tratamiento.

En esta dirección, vale la pena profundizar y discutir el desarrollo de estas funciones (sueño, dieta, lenguaje), buscando aclarar su correlación constitutiva, el vínculo entre ellas.

\section{COMIDA Y LENGUAJE: LA SORPRESA EN LA OBSERVACIÓN}

Hace algún tiempo, en la vida cotidiana de la clínica infantil, una observación de indicios de poder comenzó a cuestionarnos.

Esto ocurrió frente a las repetidas narrativas de los padres sobre los problemas de alimentación en los procedimientos de evaluación o incluso en el seguimiento de los planes terapéuticos en el caso de niños con diversos problemas de desarrollo del lenguaje. Al tratar con estos niños, tarde o temprano, surgieron quejas sobre la alimentación, pasando de la idiosincrasia a los trastornos de la deglución, y su repetición insistente fue lo que ganó lugar en nuestra escucha clínica y, con esto, el valor indicia de la observación ganó relevancia.

A partir de estas observaciones, estructuramos la investigación con una muestra de 35 pacientes, y la evidencia obtenida aclaró importantes concomitancias entre el lenguaje y los problemas alimentarios, sugiriendo una correlación significativa entre ellos (PALLADINO, CUNHA, SOUZA, 2004 y 2007).

Una lectura psicoanalítica de la pregunta, ofrecida por la Revista Francesa de Ortofonía (2004), nos permitió recubrir teóricamente los resultados de esta RC: 98234

Disponible: https://www.nucleodoconhecimento.com.br/psicologia-es/comer-y-hablar 
investigación, lo que nos lleva a pensar en la correlación entre el lenguaje y los problemas de alimentación bajo la noción de oralidad, como propone Thibaut (2006, p. 115): la zona oral es una de las zonas egénicas del cuerpo, es decir, un espacio sostenido por un en el que muchas funciones se barajan en el plano común del funcionamiento simbólico. La boca (órgano) es, en este sentido, el territorio de la comida, el lenguaje y los afectos.

En otras palabras, la zona oral es el campo somático en el que la oralidad, como plano psíquico, entrelaza simbólicamente la comida y el lenguaje.

\section{COMIDA, LENGUAJE Y SUEÑO: UNA NUEVA ARTICULACIÓN}

Este reposicionamiento conceptual dirigió y desplegó nuestra escucha clínica a otros espacios, llevándonos, más recientemente, a reelaborar la relación de la comida y el lenguaje a partir de la inclusión del sueño, formando una tríada constitutiva del niño. También se incluyó el sueño porque también es protagonista en la escena fundamental de la constitución del niño (PALLADINO, 2016, 2018). El sueño está ligado a la escena de la alimentación del bebé y forma parte de las narrativas parentales en el caso de los niños con problemas de desarrollo del lenguaje, aunque todavía opacos, es decir, considerados inútiles o con poco valor indicia en términos de riesgo para el desarrollo infantil.

Asumiendo la correlación constitutiva entre estas funciones, será necesario aclarar la alineación sintomatológica observada entre los síntomas de alimentación, sueño y lenguaje, así como analizar las posibles implicaciones en las conductas diagnósticas y de tratamiento en estos casos.

El sueño, función biológica fundamental, importante para la restauración del metabolismo energético cerebral y la consolidación de la memoria (CABALLO, NAVARRO, SIERRA, 2002), así como para el propio equilibrio psicológico, resulta de una organización gradual temporal, estructural y fisiológica del sueño. -ritmo de vigilia (GEIB, 2007; PIAULINO DE ARAÚJO, 2012.) Es un estado de funcionamiento

RC: 98234

Disponible: https://www.nucleodoconhecimento.com.br/psicologia-es/comer-y-hablar 
cerebral con dos fases diferenciadas y medibles: sueño REM (Rapid Eye Movement) y sueño NREM (No Rapid Eye Movement). Las diferencias se encuentran principalmente en términos de mecanismos metabólicos con los consiguientes cambios en los procesos fisiológicos y las condiciones posturales. En el sueño REM, hay un aumento de los niveles metabólicos, cierta atonía muscular, reducción de la temperatura corporal, ritmo respiratorio equilibrado (con pocas y breves apneas), movimientos oculares rápidos y, en el caso de los niños, pueden aparecer llantos / sonrisas / quejidos. En el sueño NREM hay una disminución de los niveles metabólicos, una disminución de la frecuencia respiratoria y la movilidad corporal (JOHN, 2000). En esta fase hay un extenso trabajo de regeneración celular y en la otra, la fase REM, principalmente de regeneración psicológica, ya que es aquí donde se desarrollan la mayoría de los sueños.

El sueño REM y NREM se alternan, así como los estados de sueño y vigilia se alternan. El ciclo entre los estados de sueño y vigilia, al principio sólo determinado biológicamente, comienza a organizarse aún en fase fetal y su constitución está estrechamente relacionada con el desarrollo del Sistema Nervioso Central (GEIB, 2007). Poco a poco, esta organización sufre el impacto de estímulos exógenos, como la luminosidad intensa, los sonidos variados $y$, sobre todo, la presencia humana, haciendo que el ciclo sueño-vigilia tenga un ritmo marcado por esta doble interferencia: endógena y exógena.

Al nacer, el patrón de sueño del bebé, esencialmente fisiológico, es más simple, con dos estados, activo (sueño REM) y tranquilo (sueño NREM), en un ritmo de sueñovigilia llamado ultradiano, aún no dominado por la alternancia día-noche. Los ciclos se alternan en un tiempo más corto, y en los primeros meses, predomina el estado activo: el bebé duerme en cualquier momento y se despierta con mucha facilidad, y la vigilia se anuncia llorando y solo más tarde se observa la vigilia tranquila. Con el tiempo, este ritmo se ve modificado por el entorno, provocando algunos atipos anárquicos, es decir, cambios al principio completamente desorganizados y desorganizando el ciclo del sueño, no solo por los estímulos luminosos y audibles,

RC: 98234

Disponible: https://www.nucleodoconhecimento.com.br/psicologia-es/comer-y-hablar 
sino principalmente por la acción humana, que acaba modulando el ritmo que, finalmente, se convierte en cicardiano, con largos periodos nocturnos de sueño tranquilo o incluso de vigilia más tranquila. (PIAULINO DE ARAÚJO 2012; JOHN, 2000)

Una acción humana prevalente en el tema del sueño de los bebés es la alimentación, por dos razones principales: a menudo precede al momento en que el bebé duerme, además del hecho de confiar en otro ser humano para su efectividad. Así, lo que al principio tiene una determinación sólo endógena, comienza a sufrir influencia del entorno externo. Aquí es donde los ritmos ultradianiano y circardiano se consolidan y comienzan a alternarse, el primero para regir la distribución de las fases del sueño y el segundo los estados de sueño y vigilia, ganando sueño por la noche y vigilia el día (PIAULINO DE ARAÚJO, 2012; JOHN, 2000).

Al tomar al bebé en su regazo para amamantarlo, la madre le ofrece un campo afectivo inigualable: el placer de escribir la leche, al escuchar las palabras, al experimentar el roce del cuerpo del otro, este deleite lo hace quedarse dormido, para darse a sí mismo.

El sueño y la comida están, por lo tanto, más allá del registro de la necesidad y, más que eso, constituyen un entrelazamiento definitivo (PENHA, 2002; FÉDIDA, 1977). Como se mencionó, estas funciones del bebé dependen de otro ser humano para su debida realización y, por esta razón, adquieren carácter simbólico, conformándose como experiencias psíquicas. Tales experiencias, en general de placer, dejan marcas psíquicas y pisadas cerebrales, creando recuerdos poderosos, que invitan a los sujetos a la convivencia con el otro.

La madre amamantará a su bebé, hablándole y/o cantándole, acariciándolo, lo que, junto con la saciedad, calma al niño y promueve el sueño. Sin embargo, el hambre, algún tiempo después, despertará al bebé, repitiendo el ciclo de vigilia-sueño.

RC: 98234

Disponible: https://www.nucleodoconhecimento.com.br/psicologia-es/comer-y-hablar 
Significa que este ciclo continuo no sólo será un efecto del ritmo orgánico sino también de un ritmo psíquico, impreso por la madre (FARIAS, 2004), a través de su cuidado.

En otras palabras, el pecho (o biberón) se convierte en un campo de intercambios y lugar de inserción del sueño (FOLINO, LOPES DE SOUZA, 2013), hablar/tararear y empacar constituyen el ethos de estas prácticas para dormir. Laznik-Penot (1997, p. 37) mostró la importancia crucial de la melopéia materna, la música de la voz de la madre que habla con el bebé. Esta escena de acalento, a su vez, implica comida, le da también existencia simbólica.

En definitiva, este ritual íntimo y repetido, que involucra cuerpo y palabras, sueño y comer, constituirá el escenario fundamental de la humanización, componiendo el plan relacional en el que se inaugura el vínculo entre el bebé y su madre. De hecho, esta perspectiva corrobora las ideas de Spinoza (2007) sobre los afectos y sus consecuencias en términos de alegría o tristeza: madre y bebé se afectan y se afectan mutuamente, lo que hace una red de conexiones afectivas que las constituyen como tales, ampliando a menudo el poder de actuar en esa relación singular y en todas las demás. El aumento del poder para actuar en el mundo es lo que Spinoza llama alegría.

Sin embargo, los afectos entre la madre y el bebé no siempre aumentan en potencia, a veces puede suceder lo contrario. Veamos un ejemplo relativamente común: las madres que pasan por baby blues (depresión benigna, transitoria, potencialmente productiva, porque forma parte de los cambios que genera la llegada de un bebé) y no encuentran la posibilidad de elaboración y aceptación por parte del contexto social que muchas veces solo reconoce el nacimiento de un niño por los sentimientos de felicidad y plenitud que, culturalmente, debe producir (FOLINO, LOPES DE SOUZA, 2013).

Estas madres deprimidas pasan menos tiempo mirando, tocando, hablando con sus bebés, mostrando menos capacidad de respuesta, espontaneidad y menores tasas

RC: 98234

Disponible: https://www.nucleodoconhecimento.com.br/psicologia-es/comer-y-hablar 
de actividad con sus bebés. La depresión posparto contribuye al efecto de que la díada se vuelva asincrónica, en la medida en que la madre es poco o no responde (SERVILHA, RAAD BUSSAB, 2015). Durante este período, el proceso afectivo entre la madre y el bebé no aumenta la potencia de ambos, al contrario, lo reduce, e incluso puede desarrollar, en algunos casos, problemas o trastornos psíquicos para las madres y para el desarrollo del bebé. La disminución del poder de acción es, a su vez, lo que Spinoza llama tristeza.

Es fundamental que el ciclo alimentación-acarcer-sueño represente experiencias psíquicas variadas para el bebé, que implican sentidos ambiguos para el bebé: la alimentación y la acarceración se convierten en espacio y tiempo de interacción madre-bebé, y el sueño de separación entre ellos - al quedarse dormido, el bebé se coloca en la cuna. Dormir representará un corte, intervalo o discontinuidad en el vínculo original entre el niño y su madre. El ritual contiene, en sí mismo, la ambigüedad entre la aceptación y la separación. Esta posición de la madre, doble, ambigua, delicada, se escenifica cuando canta y el niño se duerme para ser colocado en la cuna: acercamiento amoroso y separación.

Poco a poco, la madre espaciza los golpes -una oportunidad para consolidar el estado de vigilia- e introducir sustitutos, que se interponen entre su propio cuerpo y el del bebé como sustitutos de su presencia: chupetes, sartenes y juguetes, incluso la palabra metafórica, la que trae la madre, la representa al bebé. Estos sustitutos se insertan para operar desplazamientos en la relación fusional que conforma los primeros días del vínculo madre-bebé, son soportes para que el bebé enfrente la angustia de la separación que anuncia el sueño, la sensación de que el sueño determina la ausencia de la madre, la privación del entorno que asegura su vida y placer.

Cabe destacar que la necesidad de operar los desplazamientos para conciliar el sueño se mantiene y actualiza en la edad adulta, en los rituales que preceden a la hora de acostarse: baños, tés, lecturas... . Es como si estos rituales eliminara el

RC: 98234

Disponible: https://www.nucleodoconhecimento.com.br/psicologia-es/comer-y-hablar 
"peligro" que impone la soledad del sueño, y garantizara una especie de seguro de protección para el entumecimiento. Varias personas mayores manifiestan dificultad para conciliar el sueño por miedo a la muerte (GEIB, et al., 2003): angustia por la pérdida de uno y del mundo. El adulto tiene el funcionamiento interiorizado de la instancia materna asseguradora, operando los desplazamientos, creando los sustitutos del que fue el primer guardián del sueño, ahora inconscientemente revivido, por ejemplo, con masajes, prácticas sensuales, ingestión de alimentos, bebidas o drogas.

Durante la infancia, en cada cultura y variando debido a las condiciones sociales y económicas, hay varias formas de sortear esta angustia del sueño (tanto por parte de la madre como del bebé): cama compartida (completa o parcial), habitación compartida y la presencia de la (necesaria) lactancia nocturna (BLAIR, 2008).

Pero, es importante tener en cuenta, los médicos e investigadores tienden a referirse al Ilamado "estándar de oro" del sueño infantil en las sociedades occidentales llamadas desarrolladas, un patrón buscado temprano: cama individual, habitación separada de los padres, ausencia de alimentación nocturna.

Sin embargo, existen diferentes alertas respecto al tema del sueño, ya que también importa a los participantes de la escena y hábitos de sueño, constitutivos externos de los ciclos de sueño (GEIB, 2007). Mínimamente, se supone que para un niño que no se duerme o tiene despertares sucesivos, puede haber una madre que no piense en separar su cuerpo del cuerpo de su bebé, en dejar de hablarle, en tararear por él, en tallarlo...

Una posibilidad para superar esta angustia es el deseo del sueño, una instancia en la que lo perdido o ausente puede elevarse oniralmente.

Inicialmente, los estudios postularon que los sueños constituían una actividad exclusiva de la fase REM del sueño. Sin embargo, investigaciones más recientes muestran que no existe exclusividad, aunque existe un predominio de esta actividad

RC: 98234

Disponible: https://www.nucleodoconhecimento.com.br/psicologia-es/comer-y-hablar 
en la fase REM, con reconocimiento por parte de los estudiosos de que se pretende asegurar que el sueño realice su función regeneradora (GROMANN, 2002).

Los sueños son un efecto del entumecimiento de la censura, que impiden sensaciones, sentimientos y acciones; censura que habita en la mente de todos, perturbando tanto la vigilia como el sueño mismo. El entumecimiento da cabido a lo que se llama onírico, es decir, la construcción de pensamientos libres de interdicción. Por esta razón, ha sido que los sueños realizan restauración psíquica, tan importante como la restauración física, operada principalmente en la fase NREM.

En el desarrollo del niño, las fases del sueño se siguen gradualmente para organizar adecuadamente el sueño, convirtiéndolo en un lienzo para los sueños: desde el sueño fisiológico hasta el simbólico. El bebé necesita construir el camino entre comer, cerrar los ojos, perder a su madre (ifuera de la vista!) y reencuéntala en sueños (PENHA, 2002).

En cuanto al sueño, existe un estándar de oro para los alimentos, producidos principalmente culturalmente (incluso si se consideran aspectos orgánicos allí). En Occidente: lactancia materna durante al menos seis meses, destete natural, introducción de pastas y sólidos, un camino que, rápidamente, debe pasar de líquido caliente, de sabor indefinido, a alimentos sólidos, calientes y salados, en escenas familiares que involucran el rendimiento individual.

Sin embargo, al igual que con el tema del sueño, hay varios contenidos en juego que individualizan la escena y merecen atención (JERUSALINSKY, 2004; MADEIRA, AQUINO, 2003).

Es posible hacer un paralelismo y decir que para un niño que llora, se ahoga, rumina, vomita y/o rechaza la comida, además de posibles problemas anatomofisiológicos, puede haber una madre que tenga dificultades para posicionarse como la otra aseguradora del sujeto en constitución, realizando incorrectamente la escena alimentaria, sin alegría o incluso con tristeza.

RC: 98234

Disponible: https://www.nucleodoconhecimento.com.br/psicologia-es/comer-y-hablar 
Una escena metafórica de esta dificultad se representa en el destete, un proceso gradual que merece delicadeza, ya que se sustenta en operaciones simbólicas de separación y, por lo tanto, no es un evento "natural", obvio, cuyo suministro de alimentos y el paso de ciertos tipos a otros son siempre tranquilos.

\section{EL HILO DEL LENGUAJE}

El lenguaje es la acción humana que prevalece en los problemas de sueño y alimentación. Ambas funciones constituyen experiencias simbólicas en las palabras de la madre y en las palabras infantiles imaginadas por la madre. En la canción, en la historia, en las interjecciones de la madre y en el azúcar, en la mirada, en el cierre de ojos, en los sonidos inarticulados que son las "declaraciones" del bebé. La escena dialógica se hace como un "juego de palabras", en interpretación baktiniana. Un diálogo fundacional que persiste y marca la entrada del sujeto en el lenguaje, un territorio de encuentros y confrontaciones entre subjetividades: choque polifónico de diferentes instancias sociales, convivencia conflictiva de voces y efectos de significado. Esto sucede desde el principio, a partir de la polifonía misma del habla materna, basada en una posición ambivalente ante su bebé recién llegado.

El habla de la madre no causa un efecto directo en su bebé, sino que lo logra a través del efecto que provocó en sí misma (CORIAT, 2000), lo que hace de la relación con el bebé un campo revelador, que puede lanzarla a una experiencia de puro placer, o no. Lo que inmediatamente se centra en el bebé es la voz de la madre, esta melodía materna, dimensión musical y poética con valores afectivos (LAZNIK-PENOT, 2013), que involucra y sostiene al bebé, completamente dependiente. Melodía que crea la escena de alimentación y adormecimiento. El bebé presta especial atención a la voz de la madre y, en su ausencia, a cualquier voz melódica que tenga afectos. Así es como compone la interacción con los demás en su entorno.

Esta voz-afecto, a través de sutilezas, dinámicas y asperezas (variación de sentidos y sensaciones), es un espacio de expresión espontánea del inconsciente materno

RC: 98234

Disponible: https://www.nucleodoconhecimento.com.br/psicologia-es/comer-y-hablar 
(FÓNAGY apud LAZNIK-PENOT, 2013). Por esta razón, es importante señalar si, en este discurso melódico, se producen palabras dulces, o si, a partir de la ambivalencia materna, esta voz también puede producir, en sus ruidos, otras (palabras) muy extrañas (LAZNIK-PENOT, 2013, p. 130). Esta parece ser una forma de entender cuando la interacción no es exitosa.

La cuna melódica del discurso de la madre da la bienvenida al bebé y lo arroja a la dimensión simbólica de la existencia humana, como advierte Melgaço (2013, p.10) cuando dice que el proceso civilizador pone el poder de las palabras en el centro de atención.

A la voz de la madre se le presenta el bebé con su mirada, sus movimientos, sus sonidos, y el ir y venir entre los dos juegos de interacción, bañando con palabras la relación en la que se establecen madre y bebé.

Son tiempos constitutivos primordiales, en los que es posible tener impasses y, cuando eclosionan, síntomas, expresiones que encuentran flujo en la comida, el sueño y el lenguaje, en la medida en que estas funciones se refieren, centralmente, a la relación entre el bebé y su madre.

En esta medida y finalmente, el núcleo de la cuestión no radica ni en la madre ni en su bebé, sino en la relación entre ellos, en un proceso no de similitud, sino de identificación, marcado inicialmente por el hecho de que cuando los bebés aún no hablan, este alcance relacional se ve frustrado, sobre todo, por los procesos mentales de la madre, que sería la primera vez de la constitución de la lógica psíquica y, como señala Vorcaro (2005, p. 24), del enjambre significativo producido en el campo del Otro, en el que está inmerso el ser vivo, surge el "lugar previo" del sujeto como efecto del lenguaje. Así, lo que permanece inconsciente en la madre es más susceptible a inscripciones y revelaciones por parte del bebé (CHAVES, 2013, p. 228).

$\mathrm{RC}: 98234$

Disponible: https://www.nucleodoconhecimento.com.br/psicologia-es/comer-y-hablar 
Dormir, comer y, por qué no señalar, mirar / ser mirado, las primeras "palabras" del bebé, son el escenario de revelaciones para la madre y su bebé. En esta relación singular, el desarrollo de las funciones pilares en la vida del niño dependerá del largo camino entre la posición del objeto (de y para la madre) y la del sujeto.

\section{CONSIDERACIONES FINALES}

Como se ha dicho, este estudio surge de la clínica y, sugerimos, que debería volver a ella. Es decir, ante las quejas relacionadas con los trastornos del sueño, la alimentación y el lenguaje, es relevante que el profesional de la salud sea capaz de atender las posibles demandas derivadas de la articulación simbólica entre estas tres dimensiones; articulación inherente al funcionamiento humano cuando se asume la indisociabilidad entre lenguaje, cuerpo y psiquismo.

Si es así, tal vez el sueño también pueda incluirse en futuros estudios sobre la intrincada implicación simbólica trabajada aquí.

\section{REFERENCIAS}

ANZIEU, D. O Eu-pele. São Paulo: Casa do Psicólogo, 1989.

BLAIR, O. S. O co-leito em perspectiva. Jornal de pediatria, 84(2), 99-101, 2008.

CABALLO, V. E., NAVARRO, J. F. e SIERRA, J. C. Tratamento comportamental dos transtornos do sono In: CARLSON, N. R. Fisiologia do Comportamento. 7ed, SP: Manole, 2002.

Cabassu G. Palavras em torno do berço. In: Wanderley D. B. (org) Palavras em torno do berço. Salvador, Ágalma, 2003.

CHAVES, M. P. C. T. O lugar do analista na clínica com bebês. In: BUSNEL M. C. e MELGAÇO R. G. (orgs) $O$ bebê e as palavras: uma visão transdisciplinar sobre o bebê. Instituto Langage, 2013.

RC: 98234

Disponible: https://www.nucleodoconhecimento.com.br/psicologia-es/comer-y-hablar 
CISMARESCO, A. S. O grito neonatal e suas funções. (seção: o grito e as reações fisiológicas e emocionais das mães). In: BUSNEL, M. C. A linguagem dos bebês. São Paulo, Escuta ed., 1997.

CORIAT, E. Os flamantes bebês e a velha psicanálise. Estilos da Clínica. V.5, n.8, 2000.

FARIAS, C. N. F. e GOMES DE LIMA, G. Relação mãe-criança: esboço de um percurso na teoria psicanalítica. Revista Estilos da Clínica, ano IX, n. 16, 2004.

FÉDIDA, P. Le conte et la zone de l'endormissement. In: Corps de vide et espace de séance. Paris, Jean Pierre Delaye, 1977.

FOLINO, C. S. G. e LOPES DE SOUZA, AS. As Reverberações do encontro mãebebê: sobre a depressão e a depressividade pós-parto. In: BUSNEL, M. C. E MELGAÇO, R. G. (orgs) $O$ bebê e as palavras: uma visão transdisciplinar sobre o bebê. Instituto Langage, 2013.

GEIB, L. T. C., CATALdO NETO,A, WAINBERG R, NUNES ML. Sono e envelhecimento. Rev. Psiquiatria. Rio Gd. Sul, 25(3):453-465, 2003.

GEIB, L. T. C. Desenvolvimento dos estados do sono na infância. Revista Brasileira de Enfermagem, 60(3):323-26, 2007.

GROMANN, R. M. G. Sueño y psiquismo: el laberinto entre el cuerpo y la subjetividad. Pulsional Journal of Psychoanalysis ,164: 27-34, 2002

JERUSALINSKY, A. Psicoanálisis y desarrollo infantil. Porto alegre, Artes e Ofícios, 2004.

JUAN, M. W. Sensibilidad y especificidad y especificidad de la prueba de latencia múltiple del sueño (MSLT), la prueba de mantenimiento de la vigilia y la escala de

RC: 98234

Disponible: https://www.nucleodoconhecimento.com.br/psicologia-es/comer-y-hablar 
somnolencia de Epworth: fracaso del MSLT como estándar de oro. J. Sleep Res, 9: 5-11, 2000.

LAZNIK-PENOT, M. C. Hacia la palabra. São Paulo, Escuchar ed., 1997.

LAZNIK-PENOT, M. C. Lenguaje y comunicación del bebé de cero a tres meses. In: LAZNIK-PENOT, M. C. A hora e a vez do bebê. Instituto Langage, 2013.

LEITE, C. A. O. Quando o corpo pede um nome. Tese de doutorado, Instituto de Estudos da Linguagem, UNICAMP, 2008.

MADEIRA, I. R. e AQUINO, L. A. Problemas de abordaje difíciles: "no comer" y "no dormir". J Pediatr,79 (Supl 1): 43-54, 2003.

MELGAÇO, R. G.Prefacio. En: BUSNEL, M. C. y MELGAÇO, R. G. (organizaciones) El bebé y las palabras. São Paulo, Instituto Langage, 2013.

PALLADINO, R. R. R.; CUNHA, M.C. e SOUZA, L. A. P. Transtornos de linguagem e transtornos de alimentação em crianças. Revista Psicanálise e Universidade, 21: 95-108, 2004.

PALLADINO, R. R. R.; CUNHA, M.C. e SOUZA, L. A. P. Transtornos de linguagem e de alimentação: coincidências ou co-ocorrências? Pró-fono Revista de Atualização Científica, 19, 205-214, 2007.

PALLADINO RRR. Linguagem e sono. Anais do XXIV Congresso Brasileiro de Fonoaudiologia, Sociedade Brasileira de Fonoaudiologia, São Paulo, 2016

PALLADINO RRR. Sono e alimentação: funções psíquicas associadas. Anais do III Congresso Iberoamericano de Fonoaudiologia e XXVI Congresso Brasileiro de Fonoaudiologia. Sociedade Brasileira de Fonoaudiologia, Curitiba, 2018.

PENHA, N. C. G. Dormir nos braços da mãe: a primeira guardiã do sono. $\quad$ Rev Psichê, 6(10):65-84, 2002.

$\mathrm{RC}: 98234$

Disponible: https://www.nucleodoconhecimento.com.br/psicologia-es/comer-y-hablar 
PIAULINO DE ARAÚJO, P. D. Validação do questionário do sono infantil de Reimão e Lefèvre (QRL). Tese de doutorado, Departamento de Neurologia, USP, 2012.

RÉÉDUCATION ORTHOPHONIQUE, 44 année, juin/2006, trimestriel n. 226 - La deglutition Dysfunctionnelle, 2006.

SANTOS, M. C. Problemas alimentares da infância sem diagnóstico clínico: quando vigiar, quando atuar? Rev. Hospital de Crianças Maria Pia, vol. XIII, n.4: 342-7, 2004.

SERVILHA, B. e RAAD BUSSAB, V.S. Interação Mãe-Criança e Desenvolvimento da Linguagem: a Influência da Depressão Pós-Parto. Psico, Porto Alegre, v. 46, n. 1, pp. 101-109, jan.-mar. 2015.

SPINOZA, B. Ética. Trad. e notas de Thomaz Tadeu. Belo Horizonte, Autêntica Ed. 2007.

STORK, H.; LY, O. e MOTA. G. Os bebês falam: como você os compreende? Uma comparação intercultural. In: BUSNEL, M. C. (org) A linguagem dos bebês. Sabemos escutá-los? São Paulo: Escuta Ed., 1997.

THIBAULT, C. A língua, órgão chave das oralidades. Rééducation Orthophonique, 44 année, juin/2006, trimestriel, n.226 - La deglutition Dysfunctionnelle, p.115, 2006.

VORCARO, A. Crianças em Psicanálise, Rio de Janeiro, Companhia de Freud, 2005.

WINNICOTT, D. W. Pensando sobre crianças. Porto Alegre, Artmed, 1975.

Enviado: Septiembre de 2021.

Aprobado: Septiembre de 2021.

RC: 98234

Disponible: https://www.nucleodoconhecimento.com.br/psicologia-es/comer-y-hablar 